\title{
Supersymmetric dS/CFT
}

\section{Thomas Hertog, Gabriele Tartaglino-Mazzucchelli, Thomas Van Riet and Gerben Venken}

Institute for Theoretical Physics, KU Leuven, Celestijnenlaan 200D, 3001 Leuven, Belgium

E-mail: thomas.hertog@kuleuven.be, gabriele.tartaglino-mazzucchelli@kuleuven.be, thomas. vanriet@kuleuven. be, gerben. venken@kuleuven. be

Abstract: We put forward new explicit realisations of dS/CFT that relate $\mathcal{N}=2$ supersymmetric Euclidean vector models with reversed spin-statistics in three dimensions to specific supersymmetric Vasiliev theories in four-dimensional de Sitter space. The partition function of the free supersymmetric vector model deformed by a range of low spin deformations that preserve supersymmetry appears to specify a well-defined wave function with asymptotic de Sitter boundary conditions in the bulk. In particular we find the wave function is globally peaked at undeformed de Sitter space, with a low amplitude for strong deformations. This suggests that supersymmetric de Sitter space is stable in higher-spin gravity and in particular free from ghosts. We speculate this is a limiting case of the de Sitter realizations in exotic string theories.

KeYwords: AdS-CFT Correspondence, Extended Supersymmetry, Higher Spin Gravity

ARXIV EPRINT: 1709.06024 


\section{Contents}

1 Introduction 1

2 Supersymmetric vector models and duality 3

2.1 Free chiral superfields and higher-spin deformations 3

2.2 Spin $0 \leq s \leq 2$ deformations in components $\quad 6$

$\begin{array}{lll}2.3 \text { Duality } & 7\end{array}$

$\begin{array}{llr}3 & \text { Supersymmetric minisuperspace } & 9\end{array}$

$\begin{array}{ll}3.1 \text { Scalar deformations } & 10\end{array}$

$\begin{array}{ll}3.2 & \text { Squashings and vector fields } \\ \end{array}$

4 Speculations on a triality with exotic string theories $\quad 14$

$\begin{array}{lll}5 & \text { Discussion } & 15\end{array}$

\section{Introduction}

Gauge-gravity duality with de Sitter (dS) boundary conditions [1-3] has proved to be a fruitful route to put cosmology on firm theoretical ground. In its most ambitious and fundamental form, dS/CFT conjectures that the partition function of certain deformations of three dimensional Euclidean CFTs yields a precise formulation of the Hartle-Hawking wave function of the universe [4]. Since a wave function of the universe specifies a prior for cosmology, which in turn determines the theory's predictions for cosmological observations, this potentially provides a solid foundation for cosmology. Schematically and in the large three-volume regime the proposed dual form of the wave function reads

$$
\Psi_{H H}\left[h_{i j}, A_{s}\right]=Z_{\mathrm{QFT}}\left[\tilde{h}_{i j}, J_{s}\right] \exp \left(\mathrm{i} S_{s t}\left[h_{i j}, A_{s}\right] / \hbar\right) .
$$

Here $A_{s}$ stands for the matter configurations of spin $s$ and $h_{i j}$ is the three-geometry of the spacelike surface $\Sigma$ on which $\Psi$ is evaluated. In this paper we take the latter to be topologically a three-sphere. The sources $\left(\tilde{h}_{i j}, J_{s}\right)$ in $(1.1)$ are conformally related to the argument $\left(h_{i j}, A_{s}\right)$ of the wave function, and $S_{s t}$ are the usual surface terms.

It is a central question in holographic cosmology what class of deformed CFTs in (1.1) specifies a well-defined, normalizable wave function. Euclidean AdS/CFT provides a starting point to study this since its generalization to complex relevant deformations of CFTs implies a realisation of dS/CFT that is valid in the semiclassical approximation in Einstein gravity [5-8] and possibly exact in Vasiliev gravity in dS [9]. It has been suggested indeed that Euclidean AdS and Lorentzian dS, and their duals, can be viewed as two real domains of a single complexified theory $[1,5,10-13]$. 
In these examples of $\mathrm{dS} / \mathrm{CFT}$ the partition functions featuring in (1.1) are the inverse of those of the original AdS/CFT duals [8]. The case of higher-spin gravity is particularly illuminating because the duals are vector models for which the partition function can be evaluated explicitly for a range of deformations ${ }^{1}$ [14-17]. The Vasiliev higher-spin (HS) theory has massive scalars and an infinite tower of massless gauge fields of increasing spin [18]. The duals have conserved currents for the same symmetries [19, 20]. Deforming the boundary theory action with a conserved current $J_{s}$ corresponds to turning on the spin- $s$ field $A_{s}$ in the bulk. ${ }^{2}$ Explicit calculations of the partition function as a function of homogeneous scalar and spin-2 deformations in the $\operatorname{Sp}(N)$ vector model, which is dual to the minimal Vasiliev theory in dS [9], have provided some evidence that dS/CFT yields a well-defined wave function and in particular one which is better behaved than the usual semiclassical Hartle-Hawking wave function in Einstein gravity [21].

So far attention has focussed on the duality between the minimal Vasiliev theory in de Sitter and the dual $\operatorname{Sp}(N)$ models consisting of anti-commuting scalars. In this case the dS duality follows relatively directly from the higher-spin duality in AdS [20]. However, the connection between the AdS and the dS domain of the theories appears more general and profound $[1,10,12,13]$, which suggests there may well be a broader set of realizations of $\mathrm{dS} / \mathrm{CFT}$ in the same spirit. Here we show that the supersymmetric extension of the higherspin duality in AdS [22] also carries over to dS. This is particularly interesting because a supersymmetric realisation of dS/CFT has a better chance of specifying a well-defined wave function that predicts stable asymptotic de Sitter space. In fact Ooguri and Vafa [23] have recently advocated that supersymmetry is essential even for AdS holography, ${ }^{3}$ although they note higher-spin gravity may evade their arguments.

It is usually argued that unbroken supersymmetry and dS space do not go together (see e.g. [26]). ${ }^{4}$ This is because in $\mathrm{dS}$ space there is no positive conserved quantity, whereas supersymmetry would allow one to construct one. Indeed if there were a nonzero supercharge $Q$ then either $\left(Q+Q^{\dagger}\right)$ or i $\left(Q-Q^{\dagger}\right)$ would be Hermitian. Redefining $Q$ to be the Hermitian supercharge would then imply that $Q^{2}$ were a positive conserved quantity, which does not exist in dS.

The fact that the known dS vacua in supergravity have ghosts $[28,29]$, indicating they are perturbatively unstable, is a manifestation of this general argument. Supersymmetric HS gravity theories in de Sitter may however circumvent this problem. This is because in the higher-spin theories in dS constructed in [30] the Hermitian conjugate is an antiinvolution, defined as $\left(Q^{\dagger}\right)^{\dagger}=-Q$. With this definition one cannot construct a Hermitian quantity from a supercharge $Q$. In fact, the $\mathcal{N}=2 \mathrm{dS}_{4}$ supersymmetry algebra is realized

\footnotetext{
${ }^{1}$ This amounts to a minisuperspace approximation in cosmology. Note that we will be using the term superspace in two different contexts: on the one hand there is the minisuperspace of quantum cosmology, on the other hand we have the superspace used for supersymmetry.

${ }^{2}$ The spin-0 field carries no indices and is not really a current. Still, it is usually referred to as such.

${ }^{3}$ Related to this, Danielsson et al. [24] have conjectured that all non-supersymmetric AdS vacua are even perturbatively unstable, and in [25] it was argued that all non-supersymmetric vacua — be it AdS, Minkowski or dS - must eventually decay.

${ }^{4}$ Note that superconformal theories on a fixed de Sitter background do exist [27]. They avoid the argument of [26] by the existence of an everywhere timelike conformal killing vector.
} 
in terms of oscillators of Vasiliev theory, comprising the bosonic fields $y_{\alpha}$ and $\bar{y}_{\dot{\alpha}}=\left(y_{\alpha}\right)^{\dagger}$, such that the momentum operator $P_{a}=\mathrm{i} / 4\left(\sigma_{a}\right)^{\alpha \dot{\beta}} y_{\alpha} \bar{y}_{\dot{\beta}}$ is anti-Hermitian. Hence, from the irrepresentations of the higher-spin superalgebra there does not seem to exist an operator associated to a globally defined Hamiltonian in the first place.

In this paper we provide evidence, using holography, that supersymmetric de Sitter space is stable and has no ghosts in higher-spin gravity. We first propose a supersymmetric generalization of the higher-spin dualities in de Sitter. The bulk theories involved are the supersymmetric extensions of Vasiliev theory described in [30]. On the boundary side we construct, in section 2 , new $\mathcal{N}=2$ supersymmetric extensions of the threedimensional $\operatorname{Sp}(N)$ models. We then relate these to the theories of Sezgin and Sundel in section 2.3, thereby establishing a supersymmetric gauge-gravity duality with de Sitter boundary conditions. We also briefly discuss the fermions in the theory. In section 3 we evaluate the partition function of the supersymmetric extension of the free $\operatorname{Sp}(N)$ model as a function of homogeneous scalar, vector and spin-2 deformations that preserve supersymmetry. The duality put forward in section 2 conjectures that the partition function specifies the Hartle-Hawking wave function in a supersymmetric minisuperspace consisting of anisotropic deformations of de Sitter space with scalar and vector matter. We find the wave function is globally peaked at the undeformed de Sitter space, with a low amplitude for strong deformations. This indicates that supersymmetric de Sitter space is stable in higher-spin gravity.

It is tempting to speculate that our findings are connected to the supersymmetric dS constructions in exotic string theories [1]. The latter have vector ghosts in their supergravity limits related to the existence of non-compact $R$-symmetry groups in their representation of the algebra. ${ }^{5}$ However Hull has argued that the massive string states in exotic string theories may well render the de Sitter vacua ghost-free and unitary. In section 4 we conjecture that the supersymmetric higher-spin theories in dS that we construct are related indeed to the tensionless limit of these exotic string theories.

\section{Supersymmetric vector models and duality}

\subsection{Free chiral superfields and higher-spin deformations}

To construct Euclidian supersymmetric vector models we must consider complex fields and $\mathrm{U}(N)$ global symmetry instead of $\mathrm{O}(N)$ or $\mathrm{Sp}(N)$. The free $\mathrm{U}(N)$ vector model is a theory of $N$ commuting complex scalar fields $\phi^{i}$ and $\tilde{\phi}_{i}$, with $i=1, \ldots, N$, transforming in the fundamental and anti-fundamental representations of $\mathrm{U}(N)$ with Lagrangian $\mathcal{L}=\partial^{\mu} \tilde{\phi}_{i} \partial_{\mu} \phi^{i}$. The $\operatorname{Sp}(N)$ model or more precisely the anti-commuting $\mathrm{U}(N)$ model, also referred to as the $\mathrm{U}(-N)$ model, is defined by changing the statistics of the fields. This yields a set of complex anti-commuting scalar fields $\varphi^{i}$ and $\tilde{\varphi}_{i}$ governed by the Lagrangian $\mathcal{L}=\partial^{\mu} \tilde{\varphi}_{i} \partial_{\mu} \varphi^{i}$.

\footnotetext{
${ }^{5}$ This differs from the $\mathcal{N}=2$ Vasiliev case in [30] where the dS $R$-symmetry group is $\mathrm{SO}(2)_{R} \simeq \mathrm{U}(1)_{R}$. The $\mathcal{N}=2$ case is actually the only dS supergroup possessing compact $R$-symmetry [28, 29].
} 
Here we are interested in the three-dimensional, Euclidean $\mathcal{N}=2$ supersymmetric extension of these vector models. ${ }^{6}$ Three dimensional $\mathcal{N}=2$ supersymmetry has four real supercharges. The supersymmetric extension of a 3D scalar field can be described off-shell by using chiral and anti-chiral superfields $\Phi^{i}$ and $\tilde{\Phi}_{i}$, which we assume to be commuting for now. These satisfy the constraints $\tilde{D}_{\alpha} \Phi^{i}=0$ and $D_{\alpha} \tilde{\Phi}_{i}=0$ where the $\mathcal{N}=2$ superspace coordinates and spinor covariant derivatives are denoted $z^{M}=\left(x^{\mu}, \theta^{\alpha}, \tilde{\theta}^{\alpha}\right)$ and $\left(D_{\alpha}, \tilde{D}_{\alpha}\right){ }^{7}$ Note that in Euclidian signature $\tilde{\Phi}_{i}$ is not necessarily the complex conjugate of $\Phi^{i}$. The offshell components of the chiral superfield $\Phi^{i}$ are $\left(\phi^{i}, \psi_{\alpha}^{i}, F^{i}\right)$ with $\phi^{i}(x)=\left.\Phi^{i}\right|_{\theta=0}$ the physical complex scalar fields, $\psi_{\alpha}^{i}(x)=1 /\left.\sqrt{2} D_{\alpha} \Phi^{i}\right|_{\theta=0}$ Dirac spinors and $F^{i}(x)=-1 /\left.4 D^{2} \Phi^{i}\right|_{\theta=0}$ complex auxiliary fields (and analogously for the anti-chiral multiplet). Below we give the supersymmetry transformations for the fields in a chiral multiplet. The $\mathcal{N}=2$ supersymmetric $\mathrm{U}(N)$ model is then

$$
S=\int \mathrm{d}^{3} x \mathrm{~d}^{4} \theta \tilde{\Phi}_{i} \Phi^{i}=\int \mathrm{d}^{3} x\left[\partial_{\mu} \tilde{\phi}_{i} \partial^{\mu} \phi^{i}-\mathrm{i} \tilde{\psi}_{i} \not \partial \psi^{i}-\tilde{F}_{i} F^{i}\right]
$$

where the first term is the free bosonic $\mathrm{U}(N)$ model, the second term describes the free massless Gross-Neveu model and the last term is non-dynamical. If the $\mathrm{U}(1)_{R}$ charge and the conformal dimension of $\Phi^{i}$ are chosen to be $1 / 2$, this action is invariant under the $3 \mathrm{D}$ Euclidian superconformal group $\operatorname{OSp}(2 \mid 2,2) .{ }^{8}$ Moreover, the action possesses an infinite set of singlet higher-spin supercurrents $J^{(s)}$ [37]. The scalar supercurrent of the free theory is given by $J^{(0)}:=\tilde{\Phi}_{i} \Phi^{i}$ and satisfies on-shell the conservation equation $D^{2} J^{(0)}=\tilde{D}^{2} J^{(0)}=0$.

The superspace approach straightforwardly allows us to define an $\mathcal{N}=2$ extension of the $\mathrm{U}(-N)$ model: it suffices to replace the commuting chiral and anti-chiral superfields $\Phi^{i}$ and $\tilde{\Phi}_{i}$, with a set of anti-commuting fields $\Upsilon^{i}$ and $\tilde{\Upsilon}_{i}$. The change of statistics is such that ${ }^{9}$ $\Upsilon^{i} \Upsilon^{j}=-\Upsilon^{j} \Upsilon^{i}, \tilde{\Upsilon}_{i} \tilde{\Upsilon}_{j}=-\tilde{\Upsilon}_{j} \tilde{\Upsilon}_{i}, \Upsilon^{i} \tilde{\Upsilon}_{j}=-\tilde{\Upsilon}_{j} \Upsilon^{i}$. The anti-commuting chiral superfields have components given by the physical anti-commuting complex scalars $\varphi^{i}(x)$, commuting fermions $\chi_{\alpha}^{i}(x)$ and anti-commuting auxiliary scalar fields $G^{i}(x)$. The supersymmetry transformations of $\Upsilon^{i}$ and $\tilde{\Upsilon}_{i}$ are the same as those of $\Phi^{i}$ and $\tilde{\Phi}_{i}$. The only difference is that the component fields in the off-shell multiplet now have reversed statistics. The full superspace Lagrangian for the $\mathrm{U}(-N)$ model is then simply given by $\tilde{\Upsilon}_{i} \Upsilon^{i}$, as in (2.1). For dimension half anti-commuting chiral multiplets this has the same $\operatorname{OSp}(2 \mid 2,2)$ symmetry and higher-spin currents as the $\mathcal{N}=2 \mathrm{U}(N)$ theory.

One can extend the free $\mathrm{U}(-N)$ model to include interactions by considering an arbitrary full superspace Lagrangian ${ }^{10} K\left(\Upsilon^{i}, \tilde{\Upsilon}_{i}\right)$, and chiral and anti-chiral superpotentials

\footnotetext{
${ }^{6}$ See e.g. [22, 31-33] for an analysis of the analogous extension in the context of the AdS-Vasiliev/CFT duality.

${ }^{7}$ We adopt the notation of $[34,35]$ except for their supersymmetry parameters $(\zeta, \tilde{\zeta})$ which we will name $(\epsilon, \tilde{\epsilon})$.

${ }^{8}$ We use the $\operatorname{OSp}(2 \mid 2,2)$ supergroup notation employed in [36] which differs from the unitary quaternionic one used in $[28,29]$. In the latter case the minimal 3D Euclidian superconformal group, equivalent to the minimal $4 \mathrm{D}$ dS supergroup, is denoted as $U U_{\alpha}(1,1 ; 1 ; \mathbb{H})$.

${ }^{9}$ Note also that the commutation rules with the spinor derivatives are somewhat subtle, e.g. $D_{\alpha} \Upsilon_{i} \Upsilon_{j}=$ $\left(D_{\alpha} \Upsilon_{i}\right) \Upsilon_{j}-\Upsilon_{i} D_{\alpha} \Upsilon_{j}$

${ }^{10}$ This is the analogue of the Kähler potential for a standard supersymmetric sigma-model.
} 
$W\left(\Upsilon^{i}\right)$ and $\tilde{W}\left(\tilde{\Upsilon}_{i}\right)$. For instance an extension we will consider is the free $\mathcal{N}=4$ hypermultiplet $\mathrm{U}(-N)$ model. This is defined by a full superspace Lagrangian $\left(\tilde{\Upsilon}_{+i} \Upsilon_{+}^{i}+\tilde{\Upsilon}_{-}^{i} \Upsilon_{-i}\right)$ involving two sets of free anti-commuting chiral superfields $\Upsilon_{+}^{i}$ and $\Upsilon_{-i}$ and the antichiral cousins. By using the chiral composite $C=\Upsilon_{+}^{i} \Upsilon_{-i}$ one can construct $\mathrm{U}(N)$ invariant superpotentials $W(C)$. The quartic superpotential $\left(\Upsilon_{+}^{i} \Upsilon_{-i}\right)^{2}$ provides an example and represents a classically marginal deformation of the $\mathrm{U}(-N)$ model preserving the full $\mathcal{N}=2$ superconformal group, but not the higher-spin (HS) symmetry.

We do not elaborate further on interacting theories but concentrate first on the free $\mathcal{N}=2 \mathrm{U}(-N)$ model defined on the three sphere and deformed by background boundary sources for higher-spin supercurrents. This can be used to realize the simplest example of a supersymmetric extension of $\mathrm{dS} / \mathrm{CFT}$. In this context, the boundary sources are related to the argument of the bulk wave function in the large volume limit (cf. (1.1)).

Consider background $\mathcal{N}=2$ conformal higher-spin superfields [38] $H^{(s)}:=$ $H_{\alpha_{1} \alpha_{2} \cdots \alpha_{2 s}}(x, \theta, \tilde{\theta})$, together with the primary supercurrents of the free chiral multiplets [37], $J^{(s)}:=J_{\alpha_{1} \alpha_{2} \cdots \alpha_{2 s}}(x, \theta, \tilde{\theta})$. These are both completely symmetric tensors in the $2 s$ spinor indices. Then consider a linearly deformed full superspace Lagrangian of the form

$$
\mathcal{L}\left[H^{(s)}\right]=\tilde{\Upsilon}_{i} \Upsilon^{i}+\sum_{s=0}^{+\infty} H^{\alpha_{1} \alpha_{2} \cdots \alpha_{2 s}} J_{\alpha_{1} \alpha_{2} \cdots \alpha_{2 s}} .
$$

The deformed model is also off-shell $\operatorname{OSp}(2 \mid 2,2)$ invariant as can be proven by using the results of [38] (see also the recent $4 \mathrm{D}$ analysis of [39]). The partition function of the Lagrangian (2.2) leads to a natural superfield description of the Hartle-Hawking wave function (1.1). The spin zero supercurrent is $J^{(0)}:=\tilde{\Upsilon}_{i} \Upsilon^{i}$. For $s \geq 1$ the supercurrents satisfy on-shell the conservation equations

$$
D^{\alpha_{1}} J_{\alpha_{1} \alpha_{2} \cdots \alpha_{2 s}}=0, \quad \tilde{D}^{\alpha_{1}} J_{\alpha_{1} \alpha_{2} \cdots \alpha_{2 s}}=0, \quad \Longrightarrow \quad\left(\gamma^{\mu}\right)^{\alpha_{1} \alpha_{2}} \partial_{\mu} J_{\alpha_{1} \alpha_{2} \alpha_{3} \cdots \alpha_{2 s}}=0 .
$$

Note that the spin-1 supercurrent $[40,41]$

$$
\left.J_{\alpha \beta}=-2\left(\tilde{D}_{(\alpha} \tilde{\Upsilon}_{i}\right) D_{\beta}\right) \Upsilon^{i}+2 \mathrm{i} \tilde{\Upsilon}_{i}\left(\gamma^{\mu}\right)_{\alpha \beta} \partial_{\mu} \Upsilon^{i}-2 \mathrm{i} \Upsilon^{i}\left(\gamma^{\mu}\right)_{\alpha \beta} \partial_{\mu} \tilde{\Upsilon}_{i}
$$

contains the stress-energy tensor, the supersymmetry current and the current for the $\mathrm{U}(1)_{R}$ symmetry. Similarly, the currents $J_{\alpha_{1} \cdots \alpha_{2 s}}$ comprise several component HS currents including two series of integer and half-integer ones [37]. From here onwards we concentrate on deformations involving only the spin zero, $J^{(0)}$, and spin one, $J_{\alpha \beta}$, supercurrents.

The scalar superfield $V \equiv H^{(0)}$ describes an $\mathcal{N}=2$ vector multiplet associated with the gauging of the diagonal $\mathrm{U}(1)$ symmetry within the flavour $\mathrm{U}(N)$ group. In the context of dS/CFT the scalar components in this multiplet will be dual to the scalar and pseudoscalar currents of the bulk Vasiliev theory. On the other hand the background superfield $H_{\alpha \beta}$ describes the gravitational superfield for $3 \mathrm{D} \mathcal{N}=2$ supergravity [42]. This is the supersymmetric extension of a background metric and it contains as components the dual of the spin-two and spin-one currents in the bulk. The following action

$$
S=\int \mathrm{d}^{3} x \mathrm{~d}^{4} \theta E \tilde{\Upsilon}_{i} \mathrm{e}^{V} \Upsilon^{i},
$$


describes the nonlinear $s=0,1$ deformations of the $\mathrm{U}(-N)$ model in a simple manner as a system of anti-commuting scalar superfields in an arbitrary off-shell vector multiplet and conformal supergravity background [43, 44]. Here $E_{M}{ }^{A}$ is the supervielbein of the $3 \mathrm{D} \mathcal{N}=2$ background supergeometry and $E \equiv \operatorname{Sdet} E_{M}{ }^{A}$ is its superdeterminant. The linearization of (2.5) leads to the Lagrangian in (2.2) with $s=0,1$.

\subsection{Spin $0 \leq s \leq 2$ deformations in components}

The above superspace description is potentially abstract for many readers. We therefore give here the action in components as well as the details of the supersymmetric backgrounds used in the rest of the paper.

We consider the free chiral multiplets associated to the anti-commuting (anti-)chiral superfields $\Upsilon$ and $\tilde{\Upsilon}$. In order to study spin 0,1 and 2 deformations that preserve supersymmetry, it suffices to consider the coupling to a general background of 3D new minimal supergravity [44] in the presence of a background vector field (see also [34]). The explicit ingredients of the action (2.5) in terms of component fields are presented using the notations of $[34,35]$.

The field content of off-shell new minimal supergravity in three dimensions comprises as component fields the metric $g_{\mu \nu}$, the gravitini $\psi_{\mu}$ and $\tilde{\psi}_{\mu}$, the $\mathrm{U}(1)_{R}$ symmetry gauge field $A_{\mu}^{(R)}$, a 2-form gauge field $B_{\mu \nu}$ together with its field strength $H=\frac{\mathrm{i}}{2} \epsilon^{\mu \nu \rho} \partial_{\mu} B_{\nu \rho}$, a vector auxiliary field $C_{\mu}$ and its dual $V^{\mu}=-\mathrm{i} \epsilon^{\mu \nu \rho} \partial_{\nu} C_{\rho}$. The background Abelian vector multiplet comprise a gauge field $A_{\mu}$ together with its field strength $F_{\mu \nu}=\partial_{[\mu} A_{\nu]}$, a scalar field $\sigma$, the gaugini $\lambda$ and $\tilde{\lambda}$, and a second scalar field $D$. Note that in Euclidian signature the fields do not need to be real.

With the background fields that we consider, the off-shell supersymmetry transformations of the component fields of a chiral multiplet $\Upsilon$ of $R$-charge $q$ are:

$$
\begin{aligned}
& \delta \varphi=\sqrt{2} \epsilon \chi \\
& \delta \chi=\sqrt{2} \epsilon G-\sqrt{2} \mathrm{i} \gamma^{\mu} \tilde{\epsilon} D_{\mu} \varphi+\sqrt{2} \mathrm{i} \sigma \tilde{\epsilon} \varphi+q \sqrt{2} \mathrm{i} H \tilde{\epsilon} \varphi \\
& \delta G=-\sqrt{2} \mathrm{i} D_{\mu}\left(\tilde{\epsilon} \gamma^{\mu} \chi\right)-\sqrt{2} \mathrm{i} \sigma \tilde{\epsilon} \chi+2 \mathrm{i} \tilde{\epsilon} \tilde{\lambda} \varphi-\sqrt{2} \mathrm{i}(q-2) H \tilde{\epsilon} \chi
\end{aligned}
$$

where the $\epsilon$ and $\tilde{\epsilon}$ are the supersymmetry parameters with $\mathrm{U}(1)_{R}$ charge 1 and -1 , respectively. The covariant derivative $D_{\mu}$ are gauge and $\mathrm{U}(1)_{R}$ covariant besides including the spin connection.

We consider the case where the $\mathrm{U}(1)_{R}$ charge for the chiral supermultiplet is $q=1 / 2$. This leads to the superconformal theory with Lagrangian

$$
\mathcal{L}=D_{\mu} \tilde{\varphi} D^{\mu} \varphi+\tilde{\varphi}\left(\frac{1}{8} R+\sigma^{2}+D\right) \varphi+\mathrm{i} \tilde{\chi} \gamma^{\mu} D_{\mu} \chi+\mathrm{i} \sigma \tilde{\chi} \chi-\tilde{G} G,
$$

where $R$ is the scalar curvature of the background manifold. This is the component version of the superspace action (2.5). It is worth mentioning that the quadratic terms in the bosonic spinors $\chi$ and $\tilde{\chi}$ acquire an overall minus sign compared to the case of commuting chiral multiplets due to the unusual statistics (compare e.g. with (2.1)). Including in 
addition the chiral integral of a superpotential $W\left(\Upsilon^{I}\right)$ (where we now consider multiple chiral multiplets labelled by the index $I$ ), its component Lagrangian is

$$
\mathcal{L}_{W}=\frac{\partial W(\varphi)}{\partial \Upsilon^{J}} G^{J}+\frac{\partial^{2} W(\varphi)}{\partial \Upsilon^{J} \partial \Upsilon^{I}} \chi^{I} \chi^{J}
$$

together with its conjugate. For the interesting case with $\Upsilon_{ \pm}$multiplets mentioned earlier, the classically marginal deformation $W_{\text {marginal }}\left(\Upsilon_{ \pm}\right)=\left(\Upsilon_{+}^{i} \Upsilon_{-i}\right)^{2}$ leads to the component action

$$
\begin{aligned}
\mathcal{L}_{W_{\text {marginal }}}= & 2\left(\varphi_{+}^{k} \varphi_{-k}\right)\left(\varphi_{+}^{i} G_{-i}-\varphi_{-i} G_{+}{ }^{i}\right) \\
& +2\left(\left(\varphi_{+}^{i} \chi_{-i}+\varphi_{-i} \chi_{+}{ }^{i}\right)^{2}-2 \varphi_{+}^{i} \varphi_{-i} \chi_{+}^{j} \chi_{-j}\right) .
\end{aligned}
$$

Integrating out the auxiliary field $G_{ \pm}$from the sum of (2.7) and (2.9) we obtain the classically marginal deformation $V_{\text {marginal }}$

$$
\begin{aligned}
V_{\text {marginal }}= & 8\left(\varphi_{+}^{i} \varphi_{-i}\right)\left(\tilde{\varphi}_{+j} \tilde{\varphi}_{-}{ }^{j}\right)\left(\varphi_{+}^{k} \tilde{\varphi}_{+k}+\varphi_{-k} \tilde{\varphi}_{-}{ }^{k}\right) \\
& +2\left(\left(\varphi_{+}^{i} \chi_{-i}+\varphi_{-i} \chi_{+}{ }^{i}\right)^{2}-2 \varphi_{+}^{i} \varphi_{-i} \chi_{+}^{j} \chi_{-j}\right),
\end{aligned}
$$

where all the interaction terms are double and triple trace operators.

So far we have neglected the gaugini and gravitini which would couple to spin $1 / 2$ and $3 / 2$ currents in the actions. By truncating these modes, the invariance of the action require the supersymmetry variation of the background fermions to be zero. By imposing the variation of the gravitini to be zero, $\delta \psi_{\mu}=\delta \tilde{\psi}_{\mu}=0$, one finds the Killing spinor equations:

$$
\begin{aligned}
& \left(\nabla_{\mu}-\mathrm{i} A_{\mu}^{(R)}\right) \epsilon=-\frac{1}{2} H \gamma_{\mu} \epsilon-\mathrm{i} V_{\mu} \epsilon-\frac{1}{2} \epsilon_{\mu \nu \rho} V^{\nu} \gamma^{\rho} \epsilon, \\
& \left(\nabla_{\mu}+\mathrm{i} A_{\mu}^{(R)}\right) \tilde{\epsilon}=-\frac{1}{2} H \gamma_{\mu} \tilde{\epsilon}+\mathrm{i} V_{\mu} \tilde{\epsilon}+\frac{1}{2} \epsilon_{\mu \nu \rho} V^{\nu} \gamma^{\rho} \tilde{\epsilon} .
\end{aligned}
$$

Requiring the variation of the gaugini to be zero, $\delta \lambda=\delta \tilde{\lambda}=0$, one finds the following additional constraints on the background fields

$$
\begin{aligned}
& 0=\left(\mathrm{i}(D+\sigma H)-\frac{\mathrm{i}}{2} \epsilon^{\mu \nu \rho} \gamma_{\rho} F_{\mu \nu}-\mathrm{i} \gamma^{\mu}\left(\partial_{\mu} \sigma+\mathrm{i} V_{\mu} \sigma\right)\right) \epsilon, \\
& 0=\left(-\mathrm{i}(D+\sigma H)-\frac{\mathrm{i}}{2} \epsilon^{\mu \nu \rho} \gamma_{\rho} F_{\mu \nu}+\mathrm{i} \gamma^{\mu}\left(\partial_{\mu} \sigma-\mathrm{i} V_{\mu} \sigma\right)\right) \tilde{\epsilon} .
\end{aligned}
$$

We refer to [35] and references therein for details about the geometrical constraints imposed on a 3 -manifold admitting some residual rigid supersymmetry. The sphere $S^{3}$ of radius $l$ is a maximally supersymmetric background. In this case, besides the metric the only nontrivial field turned on is a constant $H$-flux of the form $H=\mathrm{i} / l$.

\subsection{Duality}

We conjecture that the $\mathcal{N}=2$ supersymmetric extensions of the Euclidean $\operatorname{Sp}(N)$ model that we constructed above are dual to the $\mathcal{N}=2$ supersymmetric higher-spin gravity theories in de Sitter space found in [30]. ${ }^{11}$

\footnotetext{
${ }^{11}$ We refer in particular to section 4.4 of [30] for a detailed discussion of these HS theories.
} 
There is a continuous family of Vasiliev theories in de Sitter that depend on the paritybreaking angle $\theta$. In the CFT dual, $\theta$ corresponds to a Chern-Simons coupling constant $k$, i.e. $\theta=\frac{\pi N}{2 k}$ [22]. We first consider Vasiliev theories with $\theta=0$. This corresponds to the $k \rightarrow \infty$ limit such that $N / k \rightarrow 0$. In this limit we can neglect the Chern-Simons terms since our boundary is simply connected. The dual field theory is then free and determined by the symmetry group, i.e. the amount of supersymmetry and the choice of internal gauge symmetry. Its Lagrangian is given in full generality in (2.2), and in (2.7) when restricted to low spin deformations. The symmetry groups of the bulk and boundary theories are the same: both theories have $\operatorname{OSp}(2 \mid 2,2)$ invariance possessing 8 real supercharges. They also have the same higher-spin symmetry, ${ }^{12}$ which brings the full symmetry generated by spacetime, supersymmetry and HS of both theories to $h o(1,1 \mid 4,1) \cdot{ }^{13}$

Sezgin and Sundell [30] note that the counting of dynamical fields of $\mathcal{N}=2 \mathrm{dS}_{4}$ higher spin theory matches that of $\mathcal{N}=2 \mathrm{AdS}_{4}$ higher spin theory. Since the counting of $\mathcal{N}=2$ $\mathrm{U}(N)$ currents matches the $\mathcal{N}=2 \mathrm{AdS}_{4}$ bulk fields, mutatis mutandis the counting of $\mathcal{N}=2 \mathrm{U}(-N)$ currents matches the $\mathcal{N}=2 \mathrm{dS}_{4}$ bulk fields.

The bulk theory contains in particular a scalar and a pseudo-scalar with mass $m^{2}=$ $2 / R^{2}$. This mass allows for two different boundary conditions which in the boundary theory correspond to either a free or an interacting CFT $[9,22]$. Here we consider the free boundary theory in which the $\mathrm{U}(-N)$ singlet scalar operators $\tilde{\varphi}_{i} \varphi^{i}$ have dimension $\Delta=1$ and $\tilde{\chi}_{i} \chi^{i}$ have $\Delta=2$. They correspond respectively to the bulk scalar and the pseudoscalar. This means the former must obey alternative boundary conditions for which the modes asymptotically behave as $\sim z$ whereas the latter obeys standard boundary (or quantization) conditions, with modes behaving as $\sim z^{2}$ asymptotically. ${ }^{14}$

There are also two towers of integer spin bulk fields or equivalently two towers of boundary integer spin conserved currents. One tower is constructed by applying derivatives to $\tilde{\phi}_{i} \phi^{i}$ to make traceless spin- $s$ currents. The other tower is made analogously from $\tilde{\psi}_{i} \psi^{i}$.

Finally there is one tower of strictly half-integer spin fields built by taking combinations of derivatives of $\psi_{\alpha}^{i}$ and $\tilde{\phi}_{i}$ and the conjugate. Fermionic half-integer higher-spin fields in the bulk enter as Grassmann valued arguments in the wave function ${ }^{15}$ (1.1). The general framework of supersymmetric quantum cosmology was developed long ago, see e.g. [53-55]. One might be concerned about the physical interpretation of wave functions that include Grassmann valued arguments. However, the wave function is not directly a

\footnotetext{
${ }^{12}$ The higher spin symmetry of free vector models follows from the properties of the Dirac and Laplace operators and hence does not depend on the spin-statistics of the field on which these act, see e.g. [45] and [46] for the super-Laplacian case.

${ }^{13}$ By extending the analysis of $[47,48]$ (see also $[49,50]$ ) to the supersymmetric case it may be possible to prove directly that the partition function of $(2.2)$ has $h o(1,1 \mid 4,1)$ symmetry. Along this direction, regarding the use of Noether method in superspace see the recent paper [51] for the $4 \mathrm{D} \mathcal{N}=1$ case.

${ }^{14}$ The dimension $\Delta_{ \pm}$of operators in the boundary theory is related to the mass $m^{2}$ of dual bulk scalars as $\Delta_{ \pm}=\frac{3}{2} \pm \sqrt{\frac{9}{4}-m^{2} R^{2}}$ where $R$ is the $\mathrm{dS}$ radius.

${ }^{15}$ To avoid confusion we note that, unlike in ordinary first quantised mechanics, the wave function in quantum cosmology does not explicitly depend on position, or momenta. Rather, the arguments of wave functions in quantum cosmology are configurations of matter fields and three-geometries which themselves are in general of course functions of position.
} 
physical observable. Probabilities for physical observables $\mathcal{O}$ are computed as

$$
<\Psi, \mathcal{O} \Psi>=\int \mathcal{D} h \mathcal{D} A_{s \in \mathbb{N}} \mathcal{D} A_{s \in \mathbb{N}+1 / 2} \bar{\Psi}\left(h, A_{s}\right) \mathcal{O}\left(h, A_{s}\right) \Psi\left(h, A_{s}\right) \Xi\left(h, A_{s}\right) .
$$

Here we have denoted separately and abstractly the integer and half-integer matter field configurations in the functional integral and we have inserted a density measure $\Xi$. This shows that for bosonic operators $\mathcal{O}$, since one integrates over the Grassmann valued $A_{s \in \mathbb{N}+1 / 2}$ fields, physically meaningful expectation values are ordinary numbers.

We conclude with a remark on interacting theories. We have constructed interacting supersymmetric vector models, with a potential of the form (2.10). It is natural to conjecture these play a role in a duality with supersymmetric higher-spin gravity theories in dS with the parity-breaking $\theta$ angle turned on. The analogous AdS/CFT discussion [22] shows that marginal double and triple trace interactions correspond to generalized 'designer gravity' boundary conditions [52] in the bulk. Interaction terms involving both $\varphi$ and $\chi$ will imply a relation between the asymptotic profiles of the bulk scalar and pseudo-scalar. In the context of dS/CFT this means the partition function computes the wave function in an unusual basis [14]. With $\theta$ turned on, however, also the Chern-Simons terms are important $[22,33]$. In this regard we expect extended $\mathcal{N}>2$ supersymmetric models to play an important and interesting role, in which the interplay between Chern-Simons and matter dynamics can render the marginal deformations exactly marginal. We leave a more precise and complete formulation of dS/CFT for interacting duals to future work and turn now to a first exploration of the physics of the duality relating the free theories.

\section{Supersymmetric minisuperspace}

We now evaluate the partition function of the free $\mathrm{U}(-N)$ model $(2.7)$ for a range of supersymmetry preserving bosonic deformations of the theory. We first consider homogeneous mass deformations of the theory. These correspond to turning on a supersymmetric vector multiplet background. Next we compute the theory on squashed three-sphere boundaries where we will see supersymmetry requires an additional spin-1 deformation be turned on.

Eq. (1.1) shows that dS/CFT relates the sources of the deformations in the partition function to the argument of the bulk wave function in the large three-volume limit. The dependence of the partition function on the values of the above sources therefore yields a cosmological measure on a minisuperspace of asymptotically dS configurations. From this measure predictions for semi-local observables in cosmology can be derived through further coarse-graining. The deformations we consider here specify a minisuperspace that comprises a class of anisotropic deformations of de Sitter space (which have squashed sphere future boundaries) and asymptotic dS universes with an early phase of scalar field inflation.

We consider free dual theories only in this section. Their partition functions are inversely related to the partition functions of the corresponding $\mathrm{U}(N)$ theories with ordinary spin-statistics. By Gaussian integration the latter take the form

$$
Z \propto \frac{\operatorname{det}(\text { half-integer spin field eigenvalues) }}{\operatorname{det}(\text { integer spin field eigenvalues) }}
$$


whereas for the theories with opposite spin statistics we have,

$$
Z \propto \frac{\text { det(integer spin field eigenvalues) }}{\operatorname{det}(\text { half-integer spin field eigenvalues) }} .
$$

This relation is an exact example of the more general close connection between (Euclidean) $\mathrm{AdS} / \mathrm{CFT}$ and dS/CFT evoked in the Introduction. It means that for the purpose of this section we can either work with the original free chiral multiplets (see e.g. [35]) and use (3.1) or evaluate directly (3.2). We illustrate both methods below.

Before we proceed with our analysis we should comment on two subtleties. First, in the presence of background fields contact terms can appear that render the value of the partition function unphysical [56]. A meaningful dS/CFT duality of the form (1.1) requires at least the norm of the partition function be physical, which is guaranteed if the duals are reflection positive. However we lack a proof of this in the present context. ${ }^{16} \mathrm{~A}$ second subtlety concerns the regularisation of the determinants. On the $\mathrm{U}(N)$ side the regularisation is fixed by $\mathcal{N}=2$ localisation [34]. We assume the same regularisation holds in the $\mathrm{U}(-N)$ case. Localisation for the $\mathrm{U}(-N)$ theory should parallel the result of the $\mathrm{U}(N)$ theory because the off-shell supersymmetry on the three manifolds is the same in the two cases.

\subsection{Scalar deformations}

The action of the undeformed supersymmetric $\mathrm{U}(-N)$ model $(2.7)$ on a round $S^{3}$ boundary is

$$
S_{c h i}=\int d^{3} x \sqrt{h}\left[\partial_{\mu} \tilde{\varphi}_{i} \partial^{\mu} \varphi^{i}+\frac{3}{4 l^{2}} \tilde{\varphi}_{i} \varphi^{i}+\mathrm{i} \tilde{\chi}_{i} \not \nabla \chi^{i}-\tilde{G}_{i} G^{i}\right] .
$$

We consider mass deformations of this theory which break conformal symmetry, but preserve supersymmetry ${ }^{17}$ by a coupling to the vector multiplet $V$. First we set the gauge multiplet to a BPS configuration in which only the scalar fields $\sigma$ and $D$ are nonzero and constant. The constraints (2.12) then imply that a supersymmetric vector multiplet background has $D=-\mathrm{i} \sigma / l$. Substituting this condition in (3.3) yields the following mass deformation,

$$
\mathcal{L}_{\text {mass }}=\left(\sigma^{2}-\mathrm{i} \frac{\sigma}{l}\right) \tilde{\varphi}_{i} \varphi^{i}+\mathrm{i} \sigma \tilde{\chi}_{i} \chi^{i} .
$$

where $l$ is the radius of the sphere.

We see that the deformation gives masses to both the scalars and the spinors in the boundary theory. As discussed above the component scalar current $j_{+}^{(0)}=\tilde{\varphi}_{i} \varphi^{i}$ is dual to a bulk scalar whereas the current $j_{-}^{(0)}=\tilde{\chi}_{i} \chi^{i}$ constructed out of the spinor fields is dual to the bulk pseudoscalar. Supersymmetry implies both fields are coupled.

The independent complex scalar field $\sigma$ plays the role of an external, constant mass parameter in the deformed theory [35]. It therefore enters as a source in the partition

\footnotetext{
${ }^{16}$ With more supersymmetry fewer contact terms are possible. For instance the Chern-Simons term $A \wedge d A$ is absent whenever $\mathcal{N}>2$.

${ }^{17}$ One can also consider supersymmetry breaking mass deformations by leaving $\sigma$ and $D$ in (2.7) independent. Taking $\sigma=0$ for instance yields the setup of [14] multiplied by an overall factor coming from the spinors.
} 
function. In the bulk $\sigma$ corresponds to the coefficient of the subleading term $\sim z^{2}$ in the asymptotic profile of the scalar. The phase of $\sigma$ is determined from the requirement that the theory must have a well-defined, asymptotically classical, real de Sitter structure [13]. ${ }^{18}$ For $m^{2}=2 / R^{2}$ scalars this implies in particular we must take $\sigma$ imaginary $[57,58]$. Hence the asymptotic profile of the bulk scalar has a real leading term and an imaginary subleading term, precisely what one expects for scalar fields in the Euclidean Hartle-Hawking vacuum. For the susy deformations we consider, the boundary conditions on the pseudo-scalar are completely fixed by those on the scalar. We are thus led to consider the following mass deformation $\sigma=\mathrm{i} m$, with $m$ real,

$$
\mathcal{L}_{\text {mass }}=\left[-m^{2}+\frac{m}{l}\right] \tilde{\varphi}_{i} \varphi^{i}-m \tilde{\chi}_{i} \chi^{i} .
$$

To evaluate the partition function as a function of $m$ we must first find the eigenvalues of the Laplace and Dirac operators on $S^{3}$. These are well-known and can for instance be found in [35] (see [59] for a derivation in superspace). Setting $l=1$ the scalar eigenvalues are given by

$$
\lambda_{n}=n(n+2)+3 / 4-m^{2}+m,
$$

with $n=0,1,2, \ldots$ and degeneracy $(n+1)^{2}$. Note that the first eigenvalue is negative when $m<-1 / 2$. The eigenvalues for the spinor are

$$
\lambda_{n}^{ \pm}= \pm\left(n+\frac{1}{2}\right)-m,
$$

with $n=1,2,3, \ldots$ and degeneracy $n(n+1)$. There are negative eigenvalues for all values of $m$. However, each eigenvalue has an even degeneracy. Since eigenvalues are raised to the power of their degeneracy in the partition function this means the spinor contribution need not necessarily lead to divergences.

With the eigenvalues at our disposal it is straightforward to evaluate the Gaussian integrals defining the partition function $Z[m]$. See [35] and references therein for a pedagogical description of the case with standard statistic. For a single multiplet in the theory with reversed spin-statistics we get

$$
Z[m]=\prod_{j=0}^{\infty}\left[\frac{j+m+1 / 2}{j-m+3 / 2}\right]^{j+1} .
$$

To numerically perform the product in (3.8) we found it convenient to first calculate the second derivative of the free energy. Figure $1(\mathrm{a})$ shows the resulting distribution $Z Z^{*}$ as a function of the mass parameter $m$. One sees it has a local maximum at zero deformation, corresponding to the amplitude of pure dS space in higher-spin gravity. This is in accordance with general field theory results such as the F-theorem. ${ }^{19}$ For negative

\footnotetext{
${ }^{18} \mathrm{~A}$ different but equivalent way to see this is that any other 'reality' condition on $\sigma$ would imply the bulk scalar to behave as a ghost, and the resulting wave function to be ill-defined.

${ }^{19} \mathrm{Had}$ we instead taken $\sigma$ real we would have found a pathological wave function even for small deformations. In particular the distribution would have had a $\cosh (m)$ behavior. Hence pure dS would have been a local minimum of the distribution, leading to two-point functions characteristic of an unstable ghostlike theory.
} 

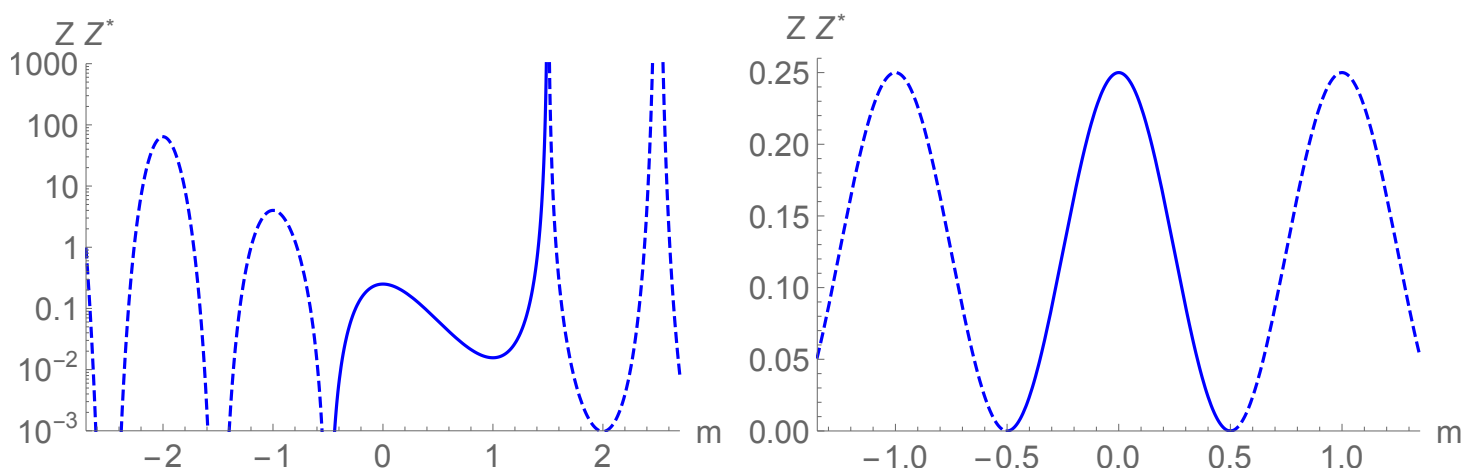

Figure 1. The response of $Z Z^{*}$ to various mass deformations for two chiral multiplets with reversed spin-statistics. We conjecture this corresponds to the Hartle-Hawking wave function in a homogeneous isotropic minisuperspace model in HS gravity. Left: both multiplets are in the same representation of the gauge multiplets inducing the mass deformation eq. (3.5) with parameter $m$. Right: the two multiplets are in opposite representations; one has mass parameter $m$ and the other $-m$ under eq. (3.5).

masses the distribution closely resembles that found in [14]. In particular it goes to zero as $m \rightarrow-1 / 2$. For more negative masses the holographic wave function is zero. This is because the path integral defining the partition function diverges in deformed (free) theories for which the scalar operator in the action has a negative eigenvalue. Hence the form (3.8) no longer holds. Therefore we show the behavior following from (3.8) with dotted curves in figure $1(\mathrm{a})$.

For positive masses the distribution diverges as $m \rightarrow 3 / 2$. Whether or not this renders the wave function non-normalizable and ill-defined may depend on the integration measure. However this behavior can be improved by adding a flavor symmetry. This can be done by placing $N$ chiral multiplets in the fundamental and $N$ in the anti-fundamental of the gauge multiplet, as for the case with $\Upsilon_{+}^{i}$ and $\Upsilon_{-i}$ described in section 2. In the BPS configuration of the gauge multiplet, this means half of the fields have opposite mass $m$. Figure 1(b) shows the resulting distribution $Z Z^{*}[m]$ which is of the form of a cosine squared. The wave function now has support on the interval $|m| \leq 1 / 2$ only. For larger values it is zero. The distribution is well-behaved over the entire configuration space and has a global maximum at $m=0$.

The addition of a flavor symmetry may appear ad hoc, but it is not. In [22], it is precisely the addition of such flavor symmetry that relates the model to string theory. Imposing this constraint from string theory in our setup appears to enhance the stability of de Sitter space.

The distribution $Z Z^{*}[m]$ specifies the no-boundary wave function $\Psi\left[A_{+}^{(0)}, A_{-}^{(0)}\right]$ in a supersymmetric minisuperspace model consisting of homogeneous, asymptotically de Sitter universes in HS gravity with a scalar $A_{+}^{(0)}$ and a pseudo-scalar $A_{-}^{(0)}$ turned on. The histories therefore exhibit an interior region with scalar field driven inflation. The upper bound on the deformation $m$ might mean the bulk potential is such that there is a maximum number of efolds of scalar field inflation. 


\subsection{Squashings and vector fields}

Next we consider deformations of the background metric which preserve four real supercharges. In particular we consider the partition function of the free $\mathrm{U}(-N)$ model $(2.7)$ on homogeneous squashings of the three-sphere that are characterized by a squashing parameter $v>0$. The metric can be written as

$$
d s^{2}=d \theta^{2}+\sin ^{2} \theta d \phi^{2}+\frac{1}{v^{2}}(d \psi+\cos \theta d \phi)^{2}
$$

where $\theta, \phi, \psi$ are the Euler angles on $S^{3}$ such that $\theta \in[0, \pi], \phi \in[0,2 \pi]$ and $\psi \in[0,4 \pi]$. Supersymmetry requires that we turn on a background $\mathrm{U}(1)_{R}$ symmetry gauge field on squashed backgrounds. In the $\mathrm{U}(N)$ model with standard spin-statistics, this is given by the one-form $[60,61]$

$$
A^{(R)}=-\frac{1}{2 v^{2}} \sqrt{1-v^{2}}(d \psi+\cos \theta d \phi) .
$$

In this section we evaluate the wave function with asymptotic dS boundary conditions using the inverse of the partition function of deformations of the $\mathrm{U}(N)$ theory. As before the phases of the sources are specified by the condition that the wave function is defined on a real configuration space that is asymptotically dS [8]. The gauge field $A^{(R)}$ is the boundary value of a bulk gauge field $\vec{A}$ in the dual AdS theory. To evaluate the wave function in the dS domain $\vec{A}$ must be asymptotically imaginary, because the original AdS scale factor $a$ is also imaginary in the dS domain. Taken together this yields asymptotically real frame fields $\vec{A} / a$ on the dS side [62]. Hence we ought to compute the partition function for purely imaginary values of the source $A^{(R)}$. This selects the range $v \geq 1$ in (3.10). Note that the round three-sphere corresponds to $v=1$. On this background, the gauge field is appropriately turned off.

The free energy of AdS dual $\mathrm{U}(N)$ theories on squashed boundaries was computed in $[35,60,61,63]$. From the relations (3.1) and (3.2) it follows that it suffices to change its overall sign to find the free energy of the dS dual theories with reversed spin-statistics. In the $v \geq 1$ domain this yields the following partition function (for a single multiplet),

$Z(v)=\operatorname{Exp}\left[-\int_{0}^{\infty} \frac{d x}{2 x}\left(\frac{\sinh (x / v)}{\sinh \left(x\left(v^{-1} \pm \sqrt{v^{-2}-1}\right)\right) \sinh \left(x /\left(v^{-1} \pm \sqrt{v^{-2}-1}\right)\right)}-\frac{1}{v x}\right)\right]$.

This specifies the large three-volume limit of the no-boundary wave function in higherspin gravity in the one-dimensional minisuperspace of anisotropic deformations of $\mathrm{dS}$ coupled to a gauge field. Figure 2 shows the resulting distribution is well-behaved and normalizable with a global maximum at pure dS space. In particular, the constraints implied by supersymmetry - together with a careful analysis of the asymptotic structure - appear to eliminate the usual problem that gauge fields in supergravity theories on de Sitter backgrounds are ghosts.

To conclude we comment on deformations that are non-supersymmetric squashings of chiral multiplets where one does not turn on a background gauge field. The free energy of such deformed theories was computed in [64] in the context of AdS duals. For free chiral 


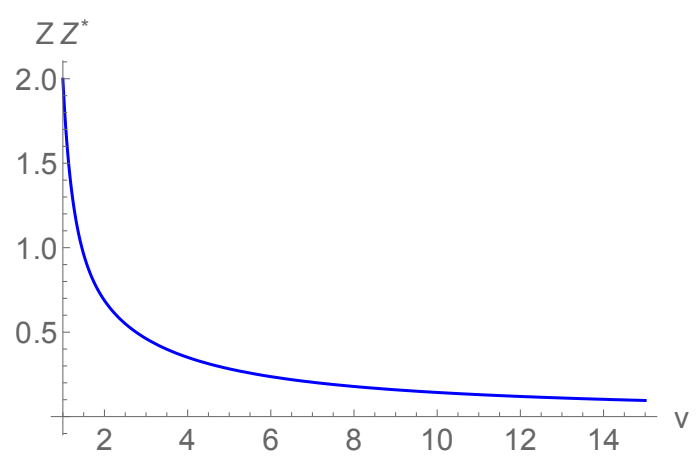

Figure 2. The holographic Hartle-Hawking wave function in higher-spin gravity in the onedimensional minisuperspace of anisotropic but homogeneous deformations of de Sitter coupled to a gauge field, parameterized by $v$, for a single multiplet.

multiplets this can be interpreted in the context of dS/CFT by inverting the partition function as we discussed. This yields a well-behaved distribution in which undeformed dS space seems stable, at least against small deformations. This indicates that for $v \geq 1$ the contributions to $Z$ due to the chiral multiplet and the background gauge field are separately stable under squashings. On the other hand, for $v<1$ there is a clear difference. The translation to $\mathrm{dS}$ of the results of [64] shows that $v=1$ is a local maximum for nonsupersymmetric squashings even when one includes the $v<1$ regime. By contrast this would not be the case if one were to extend our result for supersymmetric squashings to $v<1$, which would yield a distribution that diverges as $v \rightarrow 0$. This is precisely what one expects because under the boundary conditions corresponding to $v<1$, which we did not take, the vector field is ghost-like [62]. It is interesting that the $v<1$ regime covers the entire region of configuration space where the Yamabe invariant of the conformal boundary is negative. The fact that the holographic wave function has no support in this region once gauge fields are taken in account lends further support to the conjecture [21] that the holographic measure strongly suppresses conformal boundaries far from the round conformal structure.

\section{Speculations on a triality with exotic string theories}

In the context of ordinary AdS/CFT it has been argued [22] that a triality relates type IIA string theory on $\mathrm{AdS}_{4} \times \mathrm{CP}_{3}$ (possibly with some $B$-field flux) to specific Vasiliev theories in $\mathrm{AdS}_{4}$ and Chern-Simons vector models. These type IIA backgrounds lift to 11-dimensional supergravity on $\mathrm{AdS}_{4} \times \mathrm{S}^{7} / \mathrm{Z}_{k}$ (possibly with torsional flux added).

Our results suggest this triality can be 'Wick rotated' and thus generalized to a dS context where the third party would be the exotic M-theory $M_{9,2}^{-}$in a $\mathrm{dS}_{4} \times \mathrm{AdS}_{7} / \mathrm{Z}_{k}$ background constructed by Hull [1]. ${ }^{20}$ This exotic M-theory has two time directions and

\footnotetext{
${ }^{20}$ See [10] for this notation, where the possible existence of exotic string theories was also connected to supergroup-gaugings and negative branes. Negative branes naturally lead to signature changes between different regions of spacetime and thereby connect AdS and dS. Hence they may play a role in a microscopic description of the no-boundary wave function.
} 


\begin{tabular}{|l|llllllllll}
\hline & 0 & 1 & 2 & 3 & 4 & 5 & 6 & 7 & 8 & 9 \\
\hline$N S 5$ & $\bullet$ & $\bullet$ & $\bullet$ & $\bullet$ & $\bullet$ & $\bullet$ & & & & \\
$D 3$ & $\bullet$ & $\bullet$ & $\bullet$ & & & $\bullet$ & & \\
$D 5$ & $\bullet$ & $\bullet$ & $\bullet$ & $\bullet$ & $\bullet$ & & & & $\bullet$
\end{tabular}$\Longrightarrow \quad$\begin{tabular}{|l|llllllllll|}
\hline & \\
\hline$E N S 5$ & & $\bullet$ & $\bullet$ & $\bullet$ & $\bullet$ & $\bullet$ & & & & $\bullet$ \\
$E D 3$ & & $\bullet$ & $\bullet$ & $\bullet$ & & & $\bullet$ & & \\
$D 5$ & $\bullet$ & $\bullet$ & $\bullet$ & $\bullet$ & $\bullet$ & $\bullet$ & & & & \\
\hline
\end{tabular}

Table 1. Left table: $\mathrm{ABJ}(\mathrm{M})$ setup in IIB string theory. The Lorentzian 3D CFT is in the 012 direction. Right table: analogous setup to $\mathrm{ABJ}(\mathrm{M})$ in $\mathrm{IIB}_{(9,1)}^{-+}$with Euclidean $D 3$ branes. The Euclidean 3D CFT is in the 123 direction.

the M2 brane has a Euclidean world volume. In flat space its near-horizon geometry is $\mathrm{dS}_{4} \times \mathrm{AdS}_{7}$. The Vasiliev theories in $\mathrm{dS}$ would then be the tensionless limit of this theory.

A triality of this kind would resonate with the intuition recalled in the Introduction that exotic string theories in dS can be well-behaved despite having ghosts in the supergravity limit. Hull has argued that the massive string states render the starred string theories ghost-free [1]. The Vasiliev limit which features in the triality precisely corresponds to the limit where the massive string tower becomes massless. It is therefore plausible that Hull's 'ghost-exorcism' shows up at the classical higher-spin level. The fact that the minisuperspace wave function computed in section 3 is well behaved provides some holographic support for this.

We conclude with an illustration of how this triality might be realized more explicitly. For this purpose let us regard the above setup from a type IIB viewpoint. It is well known that ordinary $\mathrm{ABJ}(\mathrm{M})$-theory emerges in the IR from specific brane configurations (cf. table 1(a)). In table 1(b) we configure branes in an analogous manner in the exotic $\operatorname{IIB}_{(9,1)}^{-+}$ theory. The $D 3$-branes have become Euclidean while the $D 5$-branes are Lorentzian. If one can argue that strings between the Euclidean $D 3$ and the ordinary $D 5$ branes have reversed spin-statistics, then the resulting 3D field theories have limits in which they turn into the Euclidean $\mathrm{U}(-N)$ field theories we have constructed. For example, separating the Euclidean D3 and ordinary D5 branes would then correspond exactly to the mass deformation in section 3.1 with an additional flavor symmetry that gives half the fields opposite masses. $^{21}$

\section{Discussion}

We have constructed supersymmetric Euclidean vector models in three dimensions with reversed spin-statistics. We conjecture these are holographically dual to specific supersymmetric Vasiliev theories in four-dimensional de Sitter space.

We have begun to explore this duality by computing the partition function for a range of scalar, vector and tensor deformations that preserve supersymmetry. The duality asserts this specifies the Hartle-Hawking wave function in a supersymmetric minisuperspace

\footnotetext{
${ }^{21}$ As an aside we note that it was argued [65] that the near-horizon geometry of the Euclidean branes in exotic string theories does not involve de Sitter space but rather elliptic de Sitter space (where the antipodal points are identified). This in turn appears to resonate with [66] where it was shown that the physical arrow of time in asymptotic dS histories predicted by the Hartle-Hawking wave function reverses near the dS throat.
} 
consisting of anisotropic bosonic deformations of dS with scalar and vector matter. We found the wave function is globally peaked at de Sitter, with a low amplitude for strong deformations. This suggests that supersymmetric de Sitter space is stable in higher-spin gravity and in particular free from the usual ghosts.

An important generalization of our analysis concerns the calculation of the CFT partition function for deformations sourcing half-integer spin fields. Free spin- $1 / 2$ and spin- $3 / 2$ fields in de Sitter space have been studied in [67], which also derives a number of general properties of dual CFTs. In particular, [67] identifies the general form of the boundaryboundary two-point function of a bulk spinor, for an arbitrary spacetime dimension and mass, and up to a constant factor. At the time, however, no CFT dual was known to compare this with. Our model provides a concrete setup in which this can be done. The supersymmetric HS bulk theory we consider includes a specific nonlinear, interacting extension of the free spinors considered in [67]. Specializing the general result for the two-point function in [67] to the massless case in four bulk dimensions yields the following structure for the 3D boundary-boundary two-point function,

$$
<\mathcal{O}_{1 / 2}(x) \tilde{\mathcal{O}}_{1 / 2}\left(x^{\prime}\right)>=\text { const } \frac{\gamma \cdot\left(x-x^{\prime}\right)}{\left(x-x^{\prime}\right)^{4}} .
$$

This can be compared with our CFT result. On the CFT side we have, $\mathcal{O}_{1 / 2} \propto \tilde{\varphi} \chi$ and analogously for $\tilde{\mathcal{O}}_{1 / 2}$. The form of the $\mathcal{O}_{1 / 2}$ two-point function is determined up to a constant factor by conformal symmetry. Since $\mathcal{O}_{1 / 2}$ has conformal dimension $\Delta=3 / 2$, one exactly obtains (5.1). ${ }^{22}$ Therefore our CFT result matches the general structure of the fermionic two-point functions derived from a bulk analysis in [67]. This provided evidence that our proposed dS/CFT duality holds in the half-integer spin sector.

Deformations of the boundary theory that correspond to turning on spinors in the bulk are couplings of $\mathcal{O}_{1 / 2}$ and $\tilde{\mathcal{O}}_{1 / 2}$ to the background gaugini $\lambda$ and $\tilde{\lambda}$ as described in section 2.2 , although we chose to set these terms to zero. Note that the reality properties we have imposed on the background scalars $\sigma$ and $D$ imply specific reality properties on the background gaugini $\lambda$ and $\tilde{\lambda}$ through the supersymmetry variation equations. Supersymmetry thus relates the two-point function (5.1) to other two-point functions containing integer spin currents. We leave a more detailed analysis of the complete partition function in supersymmetric minisuperspace beyond the level of the two-point function, which one would expect also determines the sign of the latter, to future work.

Our results open up new ways to develop dS/CFT further. First, it would be very interesting to demonstrate the absence of ghosts directly in Vasiliev gravity in de Sitter. We have mainly focused on the duality for free theories, but it would also be interesting to formulate the duality for supersymmetric interacting theories and to clarify in particular how the Chern-Simons terms and extended $\mathcal{N}>2$ supersymmetry enter. Supersymmetry will also enable one to introduce in dS/CFT new and powerful calculational techniques, such as localisation, that have led to important advances in the context of AdS/CFT in recent years.

Finally, it would be especially interesting to understand whether the supersymmetric higher-spin theories featuring in our duality are indeed the tensionless limit of Hull's exotic

\footnotetext{
${ }^{22}$ The analogous bulk and boundary computations in the AdS/CFT case are given in [68].
} 
string theories in their de Sitter vacua. This would establish a triality similar to the one put forward in [22], which in turn may lend support to the conjecture [1] that the massive string states render the starred string theories ghost-free. This would constitute a first step toward the generalization of the HS realisations of dS/CFT to other, more realistic theories in $\mathrm{dS}$.

\section{Acknowledgments}

We thank Dionysios Anninos, Nikolay Bobev, Frederik Denef, Chris Hull, Ruben Monten, Yi Pang Antoine Van Proeyen and Yannick Vreys for helpful discussions. We furthermore thank Nikolay Bobev and Yi Pang for crucial remarks on a first draft. GV thanks Columbia University for its hospitality during part of this work. This work is supported in part by the European Research Council grant no. ERC-2013-CoG 616732 HoloQosmos, the FWO Odysseus grant G.0.E52.14N, the Interuniversity Attraction Poles Programme initiated by the Belgian Science Policy (P7/37) and the C16/16/005 grant of the KULeuven.

Open Access. This article is distributed under the terms of the Creative Commons Attribution License (CC-BY 4.0), which permits any use, distribution and reproduction in any medium, provided the original author(s) and source are credited.

\section{References}

[1] C.M. Hull, Timelike $T$ duality, de Sitter space, large- $N$ gauge theories and topological field theory, JHEP 07 (1998) 021 [hep-th/9806146] [INSPIRE].

[2] V. Balasubramanian, J. de Boer and D. Minic, Mass, entropy and holography in asymptotically de Sitter spaces, Phys. Rev. D 65 (2002) 123508 [hep-th/0110108] [INSPIRE].

[3] A. Strominger, The dS/CFT correspondence, JHEP 10 (2001) 034 [hep-th/0106113] [INSPIRE].

[4] J.B. Hartle and S.W. Hawking, Wave Function of the Universe, Phys. Rev. D 28 (1983) 2960 [INSPIRE].

[5] J.M. Maldacena, Non-Gaussian features of primordial fluctuations in single field inflationary models, JHEP 05 (2003) 013 [astro-ph/0210603] [INSPIRE].

[6] D. Harlow and D. Stanford, Operator Dictionaries and Wave Functions in AdS/CFT and $d S / C F T$, arXiv:1104.2621 [INSPIRE].

[7] J. Maldacena, Einstein Gravity from Conformal Gravity, arXiv:1105.5632 [INSPIRE].

[8] T. Hertog and J. Hartle, Holographic No-Boundary Measure, JHEP 05 (2012) 095 [arXiv:1111.6090] [INSPIRE].

[9] D. Anninos, T. Hartman and A. Strominger, Higher Spin Realization of the $d S / C F T$ Correspondence, Class. Quant. Grav. 34 (2017) 015009 [arXiv:1108.5735] [InSPIRE].

[10] R. Dijkgraaf, B. Heidenreich, P. Jefferson and C. Vafa, Negative Branes, Supergroups and the Signature of Spacetime, arXiv:1603.05665 [INSPIRE].

[11] K. Skenderis, P.K. Townsend and A. Van Proeyen, Domain-wall/cosmology correspondence in AdS/dS supergravity, JHEP 08 (2007) 036 [arXiv:0704.3918] [INSPIRE]. 
[12] E.A. Bergshoeff, J. Hartong, A. Ploegh, J. Rosseel and D. Van den Bleeken, Pseudo-supersymmetry and a tale of alternate realities, JHEP 07 (2007) 067 [arXiv:0704.3559] [INSPIRE].

[13] J.B. Hartle, S.W. Hawking and T. Hertog, Quantum Probabilities for Inflation from Holography, JCAP 01 (2014) 015 [arXiv:1207.6653] [INSPIRE].

[14] D. Anninos, F. Denef and D. Harlow, Wave function of Vasiliev's universe: a few slices thereof, Phys. Rev. D 88 (2013) 084049 [arXiv:1207.5517] [INSPIRE].

[15] D. Anninos, F. Denef, G. Konstantinidis and E. Shaghoulian, Higher Spin de Sitter Holography from Functional Determinants, JHEP 02 (2014) 007 [arXiv: 1305.6321] [INSPIRE].

[16] N. Bobev, T. Hertog and Y. Vreys, The NUTs and Bolts of Squashed Holography, JHEP 11 (2016) 140 [arXiv:1610.01497] [INSPIRE].

[17] G. Conti, T. Hertog and Y. Vreys, Holographic Measure on Eternal Inflation, arXiv: 1707.09663 [INSPIRE].

[18] M.A. Vasiliev, Consistent equation for interacting gauge fields of all spins in (3+1)-dimensions, Phys. Lett. B 243 (1990) 378 [InSPIRE].

[19] I.R. Klebanov and A.M. Polyakov, AdS dual of the critical $\mathrm{O}(N)$ vector model, Phys. Lett. B 550 (2002) 213 [hep-th/0210114] [INSPIRE].

[20] S. Giombi, Higher Spin - CFT Duality, in TASI lectures 2015, Boulder U.S.A. (2015), pg. 137 [arXiv: 1607.02967] [INSPIRE].

[21] S.W. Hawking and T. Hertog, A Smooth Exit from Eternal Inflation, arXiv:1707.07702 [INSPIRE].

[22] C.-M. Chang, S. Minwalla, T. Sharma and X. Yin, ABJ Triality: from Higher Spin Fields to Strings, J. Phys. A 46 (2013) 214009 [arXiv:1207.4485] [InSPIRE].

[23] H. Ooguri and C. Vafa, Non-supersymmetric AdS and the Swampland, arXiv:1610.01533 [INSPIRE].

[24] U. Danielsson and G. Dibitetto, Fate of stringy AdS vacua and the weak gravity conjecture, Phys. Rev. D 96 (2017) 026020 [arXiv:1611.01395] [INSPIRE].

[25] B. Freivogel and M. Kleban, Vacua Morghulis, arXiv:1610.04564 [INSPIRE].

[26] E. Witten, Quantum gravity in de Sitter space, in Strings 2001: International Conference, Mumbai India (2001) [hep-th/0106109] [INSPIRE].

[27] T. Anous, D.Z. Freedman and A. Maloney, de Sitter Supersymmetry Revisited, JHEP 07 (2014) 119 [arXiv:1403.5038] [INSPIRE].

[28] K. Pilch, P. van Nieuwenhuizen and M.F. Sohnius, de Sitter Superalgebras and Supergravity, Commun. Math. Phys. 98 (1985) 105 [InSPIRE].

[29] J. Lukierski and A. Nowicki, All Possible De Sitter Superalgebras and the Presence of Ghosts, Phys. Lett. B 151 (1985) 382.

[30] E. Sezgin and P. Sundell, Supersymmetric Higher Spin Theories, J. Phys. A 46 (2013) 214022 [arXiv: 1208.6019] [INSPIRE].

[31] R.G. Leigh and A.C. Petkou, Holography of the $N=1$ higher spin theory on AdS 4 , JHEP 06 (2003) 011 [hep-th/0304217] [INSPIRE]. 
[32] Y. Hikida and T. Wada, Marginal deformations of $3 d$ supersymmetric $\mathrm{U}(N)$ model and broken higher spin symmetry, JHEP 03 (2017) 047 [arXiv: 1701.03563] [INSPIRE].

[33] M. Honda, Y. Pang and Y. Zhu, ABJ Quadrality, JHEP 11 (2017) 190 [arXiv:1708.08472] [INSPIRE].

[34] C. Closset, T.T. Dumitrescu, G. Festuccia and Z. Komargodski, Supersymmetric Field Theories on Three-Manifolds, JHEP 05 (2013) 017 [arXiv: 1212.3388] [INSPIRE].

[35] B. Willett, Localization on three-dimensional manifolds, J. Phys. A 50 (2017) 443006 [arXiv: 1608.02958] [INSPIRE].

[36] S.M. Kuzenko and D. Sorokin, Superconformal structures on the three-sphere, JHEP 10 (2014) 80 [arXiv: 1406.7090] [INSPIRE].

[37] A.A. Nizami, T. Sharma and V. Umesh, Superspace formulation and correlation functions of $3 d$ superconformal field theories, JHEP 07 (2014) 022 [arXiv: 1308.4778] [INSPIRE].

[38] S.M. Kuzenko and D.X. Ogburn, Off-shell higher spin $N=2$ supermultiplets in three dimensions, Phys. Rev. D 94 (2016) 106010 [arXiv:1603.04668] [INSPIRE].

[39] S.M. Kuzenko, R. Manvelyan and S. Theisen, Off-shell superconformal higher spin multiplets in four dimensions, JHEP 07 (2017) 034 [arXiv: 1701.00682] [INSPIRE].

[40] T.T. Dumitrescu and N. Seiberg, Supercurrents and Brane Currents in Diverse Dimensions, JHEP 07 (2011) 095 [arXiv: 1106.0031] [INSPIRE].

[41] S.M. Kuzenko and G. Tartaglino-Mazzucchelli, Three-dimensional $N=2$ (AdS) supergravity and associated supercurrents, JHEP 12 (2011) 052 [arXiv:1109.0496] [INSPIRE].

[42] S.M. Kuzenko, Prepotentials for $N=2$ conformal supergravity in three dimensions, JHEP 12 (2012) 021 [arXiv: 1209.3894] [INSPIRE].

[43] P.S. Howe, J.M. Izquierdo, G. Papadopoulos and P.K. Townsend, New supergravities with central charges and Killing spinors in (2+1)-dimensions, Nucl. Phys. B 467 (1996) 183 [hep-th/9505032] [INSPIRE].

[44] S.M. Kuzenko, U. Lindström and G. Tartaglino-Mazzucchelli, Off-shell supergravity-matter couplings in three dimensions, JHEP 03 (2011) 120 [arXiv:1101.4013] [INSPIRE].

[45] M.G. Eastwood, Higher symmetries of the Laplacian, Annals Math. 161 (2005) 1645 [hep-th/0206233] [INSPIRE].

[46] P.S. Howe and U. Lindström, Super-Laplacians and their symmetries, JHEP 05 (2017) 119 [arXiv: 1612.06787] [INSPIRE].

[47] X. Bekaert, E. Joung and J. Mourad, On higher spin interactions with matter, JHEP 05 (2009) 126 [arXiv:0903.3338] [INSPIRE].

[48] X. Bekaert, E. Joung and J. Mourad, Effective action in a higher-spin background, JHEP 02 (2011) 048 [arXiv: 1012.2103] [INSPIRE].

[49] A.A. Tseytlin, On limits of superstring in $A d S_{5} \times S^{5}$, Theor. Math. Phys. 133 (2002) 1376 [Teor. Mat. Fiz. 133 (2002) 69] [hep-th/0201112] [INSPIRE].

[50] A.Y. Segal, Conformal higher spin theory, Nucl. Phys. B 664 (2003) 59 [hep-th/0207212] [INSPIRE]. 
[51] I.L. Buchbinder, S.J. Gates and K. Koutrolikos, Higher Spin Superfield interactions with the Chiral Supermultiplet: Conserved Supercurrents and Cubic Vertices, Universe 4 (2018) 6 [arXiv: 1708.06262] [INSPIRE].

[52] T. Hertog and G.T. Horowitz, Designer gravity and field theory effective potentials, Phys. Rev. Lett. 94 (2005) 221301 [hep-th/0412169] [INSPIRE].

[53] P.D. D'Eath and J.J. Halliwell, Fermions in Quantum Cosmology, Phys. Rev. D 35 (1987) 1100 [INSPIRE].

[54] P.D. D'Eath and D.I. Hughes, Supersymmetric minisuperspace, Phys. Lett. B 214 (1988) 498 [INSPIRE].

[55] P.D. D'Eath and D.I. Hughes, Minisuperspace with local supersymmetry, Nucl. Phys. B 378 (1992) 381 [INSPIRE].

[56] C. Closset, T.T. Dumitrescu, G. Festuccia, Z. Komargodski and N. Seiberg, Comments on Chern-Simons Contact Terms in Three Dimensions, JHEP 09 (2012) 091 [arXiv: 1206.5218] [INSPIRE].

[57] D. Anninos, T. Anous, D.Z. Freedman and G. Konstantinidis, Late-time Structure of the Bunch-Davies de Sitter Wavefunction, JCAP 11 (2015) 048 [arXiv:1406.5490] [INSPIRE].

[58] T. Hertog, R. Monten and Y. Vreys, Lorentzian Condition in Holographic Cosmology, JHEP 01 (2017) 060 [arXiv:1607.07471] [INSPIRE].

[59] I.B. Samsonov and D. Sorokin, Superfield theories on $S^{3}$ and their localization, JHEP 04 (2014) 102 [arXiv:1401.7952] [INSPIRE].

[60] Y. Imamura and D. Yokoyama, $N=2$ supersymmetric theories on squashed three-sphere, Phys. Rev. D 85 (2012) 025015 [arXiv: 1109.4734] [InSPIRE].

[61] D. Martelli and A. Passias, The gravity dual of supersymmetric gauge theories on a two-parameter deformed three-sphere, Nucl. Phys. B 877 (2013) 51 [arXiv:1306.3893] [INSPIRE].

[62] J.B. Hartle, S.W. Hawking and T. Hertog, Vector Fields in Holographic Cosmology, JHEP 11 (2013) 201 [arXiv: 1305.7190] [INSPIRE].

[63] T. Nishioka and K. Yonekura, On RG Flow of $\tau_{R R}$ for Supersymmetric Field Theories in Three-Dimensions, JHEP 05 (2013) 165 [arXiv:1303.1522] [INSPIRE].

[64] N. Bobev, P. Bueno and Y. Vreys, Comments on Squashed-sphere Partition Functions, JHEP 07 (2017) 093 [arXiv: 1705. 00292] [InSPIRE].

[65] M.K. Parikh, I. Savonije and E.P. Verlinde, Elliptic de Sitter space: dS/Z(2), Phys. Rev. D 67 (2003) 064005 [hep-th/0209120] [INSPIRE].

[66] J. Hartle and T. Hertog, Arrows of Time in the Bouncing Universes of the No-boundary Quantum State, Phys. Rev. D 85 (2012) 103524 [arXiv:1104.1733] [InSPIRE].

[67] L. Anguelova and P. Langfelder, Massive gravitino propagator in maximally symmetric spaces and fermions in dS/CFT, JHEP 03 (2003) 057 [hep-th/0302087] [INSPIRE].

[68] M. Henningson and K. Sfetsos, Spinors and the AdS/CFT correspondence, Phys. Lett. B 431 (1998) 63 [hep-th/9803251] [INSPIRE]. 